\title{
М.Ю. Пустоветов
}

\section{Устройство питания вспомогательных цепей электровоза постоянного тока: схема силовой части, методы формирования выходного напряжения}

\begin{abstract}
Приведен краткий анализ схем полупроводниковых преобразователей, используемых для питания вспомогательных цепей электровозов постоянного тока. Предложена схема силовой части устройства питания вспомогательных цепей электровоза постоянного тока, отличная от получивших распространение. Методами математического моделирования на ЭВМ проведено исследование характеристик устройства в статическом режиме. Устройство содержит емкостный делитель постоянного напряжения, автономный инвертор напряжения, трехфазный трансформатор со специфической схемой соединения обмоток III/Y. Проведено сравнительное рассмотрение энергетических характеристик устройства при разных методах формирования регулируемого напряжения на выходе инвертора с использованием широтно-импульсной модуляции. Учитывались использование напряжения контактной сети, КПД, гармонический состав токов и напряжений, значения суммарных коэффициентов гармонических составляющих, коэффициент мощности. Ожидается снижение стоимости комплекта силовых ключей преобразователя до 1,5-2,0 раза по сравнению с известными техническими решениями.

Ключевые слова: автономный инвертор напряжения, трехфазный трансформатор, электровоз постоянного тока, питание вспомогательных цепей, широтно-импульсная модуляция, гармонический состав.

doi: $10.21293 / 1818-0442-2017-20-1-136-140$
\end{abstract}

В настоящее время на борту магистральных электровозов весьма распространены потребители трёхфазного и однофазного переменного напряжения. Одной из задач, решаемых при разработке устройств для питания собственных нужд электровоза, является выбор способа преобразования высокого напряжения контактной сети в низкое трёхфазное переменное напряжение. Некоторые схемные решения для электровозов постоянного тока на базе двухуровневых и трехуровневых мостовых автономных инверторов напряжения (АИН), включая устройства с использованием трёхфазных трансформаторов (Т), опубликованы отечественными и зарубежными специалистами [1-3]. Они обладают рядом недостатков. Использование мостовой схемы двухуровневого АИН является вариантом с минимальным числом полупроводниковых ключей (6 штук) и наиболее высокой надёжностью, но требует при напряжении контактной сети 3 кВ использования транзисторов с рабочим напряжением 6,5 кВ, которые дороги.

Особенности формирования выходного напряжения в двухуровневом трехфазном мостовом АИН для случая, когда заземлена минусовая клемма входного источника постоянного напряжения, характерного для электрифицированного железнодорожного транспорта, рассмотрены в [4]: при открытом верхнем транзисторе фазы на выходе этой фазы АИН относительно земли возникает потенциал, равный полному постоянному напряжению на входе АИН. Использование трёхуровневого мостового АИН позволяет ограничиться более дешёвыми силовыми транзисторами с величиной рабочего напряжения 3,3 кВ. При этом удваивается их количество, усложняются схема и алгоритмы управления АИН.

Целью настоящей работы является предложить вариант устройства питания вспомогательных цепей электровоза постоянного тока с трёхфазным Т и АИН, позволяющий использовать минимальное количество относительно низковольтных силовых транзисторов при простой схеме соединения.

Схема силовой части устройства

Ввиду компактности и дешевизны [5] целесообразно использовать не трёхфазную группу однофазных T, а Т с единым магнитопроводом, например трехстержневым. Предлагаемая к использованию схема АИН предполагает независимое (без электрических соединений друг с другом, по открытой схеме) подключение фаз первичной обмотки Т (рис. 1).

Устройство на рис. 1 преобразует постоянное напряжение контактной сети в трёхфазное переменное напряжение, частота и величина которого могут регулироваться. Каждая фаза первичной обмотки трёхфазного Т подключена к ячейке преобразователя, представляющей собой однофазный полумостовой АИН. Например, фаза $A$ подключена к ячейке, включающей в себя транзисторные ключи $V T 1$ и $V T 2$, диоды $V D 1$ и $V D 2$, конденсаторы $C 1$ и $C 2$. Транзисторы VT1 и VT2 открываются поочерёдно на равные промежутки времени, формируя переменное напряжение на фазе $A$ первичной обмотки Т. Последовательно соединённые конденсаторы $C 7, C 8$ и $C 9$ представляют собой ёмкостный делитель, разделяющий входное постоянное напряжение контактной сети $U_{d}$ на три равные части (по количеству фаз Т и ячеек преобразователя). Пары транзисторов в остальных двух фазах работают так же, как и в фазе $A$, но со сдвигом во времени на $120^{\circ}$ эл. и $240^{\circ}$ эл., образуя на обмотках Т трёхфазную симметричную систему питающих напряжений.

Методы формирования выходного напряжения инвертора

Сравним варианты управления ключами АИН методами прямоугольной и синусоидальной (терми- 
нология согласно [6]) широтно-импульсной модуляции (ШИМ) при условии получения максимально возможного выходного напряжения АИН. На рис. 2 показаны методы осуществления ШИМ напряжения фазы: а) - прямоугольная ШИМ (метод 1), б) - прямоугольная ШИМ при форме сигнала модулирующего напряжения «меандр с паузой» с шириной им- пульса $120^{\circ}$ эл. (метод 2), в) синусоидальная ШИМ (методы 3 и 4). В качестве метода 4 используем синусоидальную ШИМ с предмодуляцией третьей гармоникой, имеющей амплитуду 0,167 от первой гармоники и перемодуляцией первой гармоники в $\pi / 2$ раз [7]. Вводимая третья гармоника синфазна с первой.

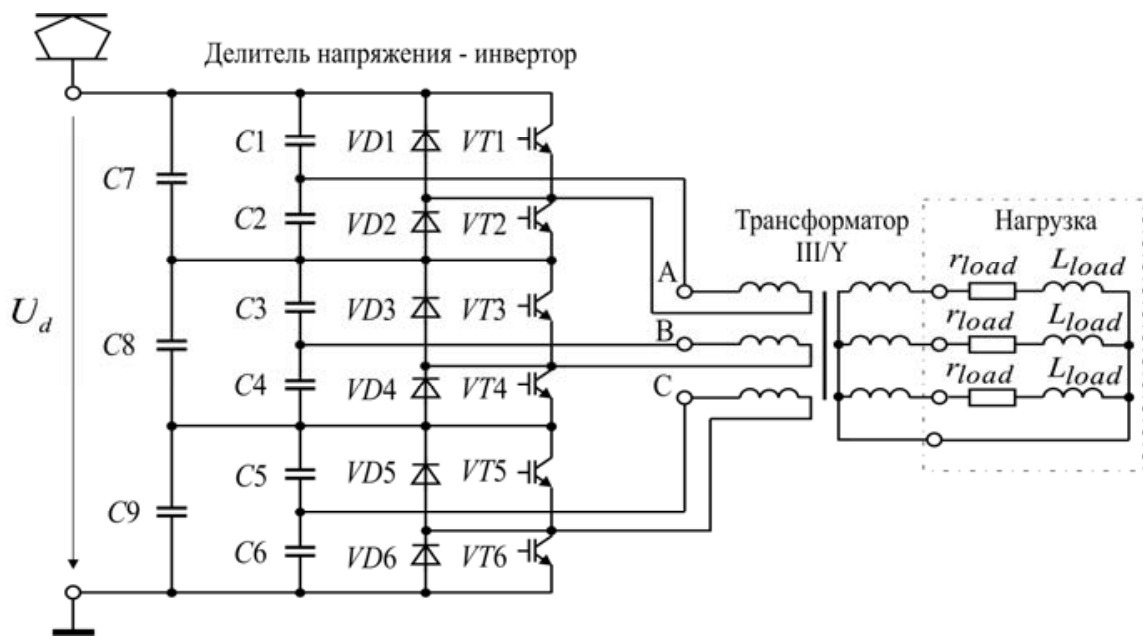

Рис. 1. Электрическая принципиальная схема устройства питания вспомогательных цепей электровоза постоянного тока
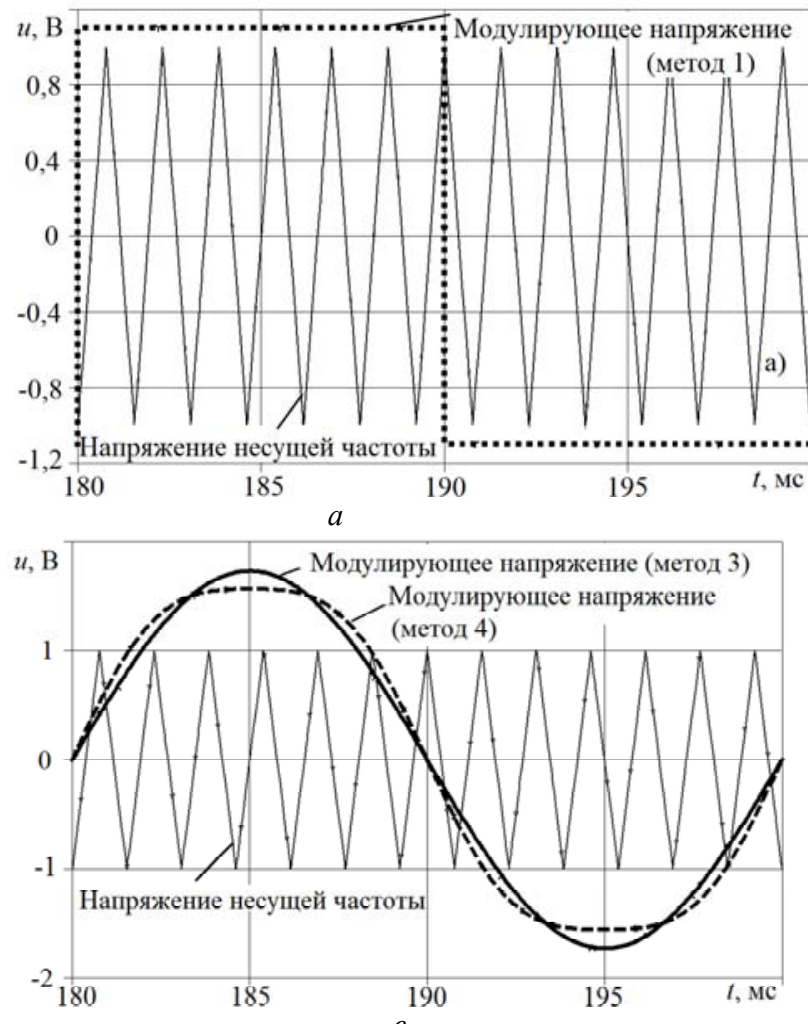

Рис. 2. Методы осуществления ШИМ напряжения фазы

При анализе режимов работы устройства питания вспомогательных цепей электровоза воспользуемся математической моделью трехфазного Т согласно $[8,9]$. Результаты компьютерного моделирования средствами PSpice [10] для сравнения методов управления преобразователем сведены в табл. 1. При моделировании приняты следующие допущения: не учитывается нелинейность кривой намагничивания

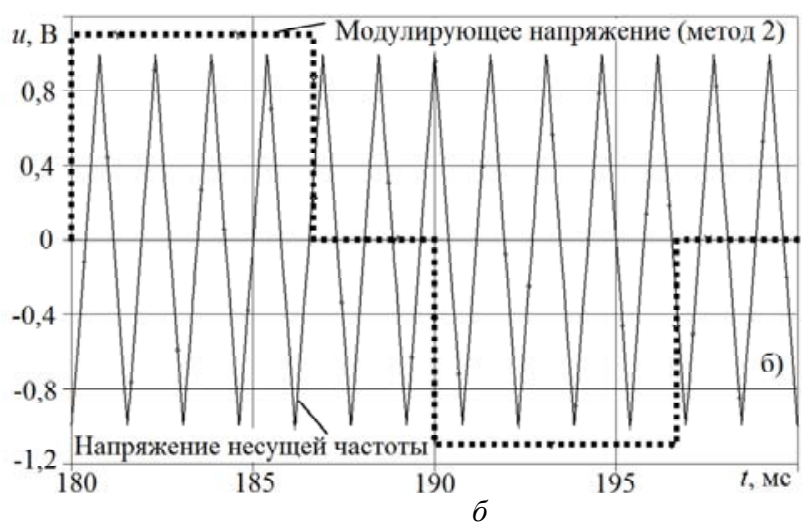

T, вместо транзисторов используются идеализированные управляемые напряжением ключи. Во всех случаях параметры Т и нагрузки одинаковы. Нагрузка симметрична с $\cos \varphi=0,88$. Частота модулирующего напряжения 50 Гц, $U_{d}=3300$ В. Все значения величин в табл. 1 представлены в относительных единицах. Мгновенное значение напряжения на одном транзисторе не превышало 1220 В.

При любом из рассмотренных методов невелики гармоники с порядками, кратными трём, в фазном и линейном напряжениях вторичной обмотки Т.

Это положительно сказывается на гармоническом составе тока вторичной обмотки. Потенциал нейтральной точки нагрузки (см. рис. 1) близок к нулю.

С точки зрения гармонического состава токов и напряжений, формируемых преобразователем, предпочтительно, когда на периоде модулирующего напряжения укладывается нечётное количество периодов напряжения несущей частоты. В противном случае токи и напряжения будут содержать четные гар- 
моники (по данным табл. 1, это слабо влияет на энергетические характеристики электротехнической системы). Метод 2 позволяет радикально уменьшить величину тока 3-й гармоники в первичной обмотке Т (до 11,09\% от 1-й гармоники). При методе 4: 3-я гармоника тока в первичной обмотке Т составляет $150,62 \%$ от 1-й, что ниже, чем при методе 1 , где $212,76 \%$.

Т а б л и ц а 1

Сравнительные результаты расчёта характеристик устройства для питания трёхфазных и однофазных вспомогательных цепей электровоза постоянного тока при различных методах формирования выходного напряжения АИН

\begin{tabular}{|c|c|c|c|c|c|c|}
\hline $\begin{array}{c}\text { Наименова- } \\
\text { ние характе- } \\
\text { ристик } \\
\end{array}$ & $\begin{array}{c}\text { Напряжение } \\
\text { 1-й гармоники фазы } \\
\text { первичной обмотки T }\end{array}$ & $\begin{array}{c}\text { Активная } \\
\text { мощность на } \\
\text { выходе T } \\
\end{array}$ & $\begin{array}{c}\text { Коэффициент } \\
\text { мощности } \\
\text { на входе Т } \\
\end{array}$ & КПД Т & $\eta_{\mathrm{T}} \cdot \cos \varphi_{1}$ & - \\
\hline $\begin{array}{c}\text { Условное } \\
\text { обозначение }\end{array}$ & $\stackrel{*}{U}_{1 \phi 1}$ & ${ }^{*} P_{2}$ & $\cos \varphi_{1}$ & $\eta_{\mathrm{T}}$ & $K_{E}$ & $K_{E} \cdot U_{1 \phi 1}^{*}$ \\
\hline
\end{tabular}

При частоте несущего напряжения 650 Гц

(13 периодов несущего напряжения ШИМ на одном периоде модулирующего)

\begin{tabular}{|c|c|c|c|c|c|c|}
\hline Метод 1 & $1,000(520,9$ В) & $1,000(73,2$ кВт) & 0,845 & 0,917 & 0,775 & 0,775 \\
\hline Метод 2 & 0,859 & 0,759 & 0,829 & 0,970 & 0,804 & 0,691 \\
\hline Метод 3 & 0,946 & 0,877 & 0,828 & 0,962 & 0,797 & 0,754 \\
\hline Метод 4 & 0,969 & 0,925 & 0,833 & 0,939 & 0,782 & 0,758 \\
\hline \multicolumn{7}{|c|}{ При частоте несущего напряжения 600 Гц } \\
\hline Метод 1 & 1 цериодов несущего напряжения ШИМ на одном периоде модулирующего) \\
\hline Метод 2 & $1,000(518,7 \mathrm{~B})$ & $1,000(73$ кВт) & 0,847 & 0,910 & 0,771 & 0,771 \\
\hline Метод 4 & 0,873 & 0,761 & 0,834 & 0,966 & 0,806 & 0,704 \\
\hline
\end{tabular}
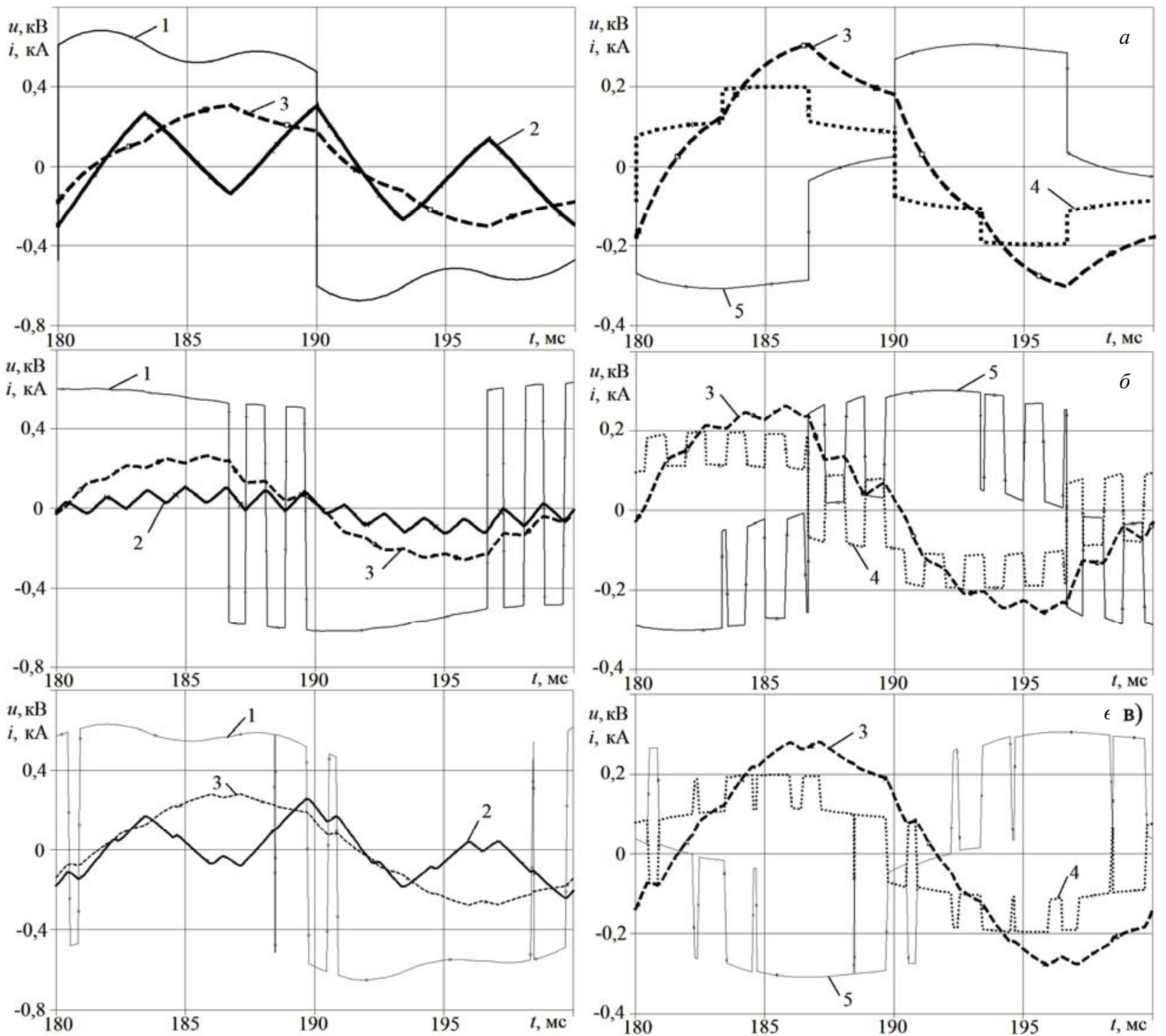

Рис. 3. Напряжения и токи Т при различных методах осуществления ШИМ напряжения фазы АИН 
Расчётные кривые напряжений и токов показаны на рис. $3: a$-метод $1 ; \sigma$ - метод $2 ;$ в - метод 4. Кривые: 1 - напряжение фазы первичной обмотки Т $U_{1 \phi} ; 2$ - ток фазы первичной обмотки Т $I_{1 \phi} ; 3$ - ток фазы вторичной обмотки Т $I_{2 \phi} ; 4$ - напряжение фазы вторичной обмотки Т $U_{2 \phi} ; 5$ - инвертированное по знаку линейное напряжение вторичной обмотки Т $U_{2 л}$. Кривые по методу 3 близки к изображенным на рис. 3, в).

Т а б ли ц а 2

Расчетный гармонический состав напряжений и токов при разных методах осуществления ШИМ напряжения фазы АИН

\begin{tabular}{|c|c|c|c|c|c|c|c|c|c|c|}
\hline Метод & 1 & 2 & 1 & 2 & 1 & 2 & 1 & 2 & 1 & 2 \\
\hline $\begin{array}{c}\text { Порядок } \\
\text { гармоники } k\end{array}$ & \multicolumn{2}{|c|}{$U_{1 \phi k}, \%$} & \multicolumn{2}{|c|}{$I_{1 \phi k}, \%$} & \multicolumn{2}{|c|}{$U_{2 \phi k}, \%$} & \multicolumn{2}{|c|}{$I_{2 \phi k}, \%$} & \multicolumn{2}{|c|}{$U_{2 л k}, \%$} \\
\hline 1 & 100,000 & 100,000 & 100,000 & 100,000 & 100,000 & 100,000 & 100,000 & 100,000 & 100,000 & 100,000 \\
\hline 3 & 39,766 & 6,274 & 212,764 & 11,090 & 0,448 & 5,072 & 0,190 & 2,804 & 0,560 & 7,510 \\
\hline 5 & 18,508 & 23,791 & 5,371 & 5,818 & 16,742 & 21,978 & 6,585 & 8,643 & 16,823 & 15,231 \\
\hline 7 & 13,485 & 11,592 & 2,801 & 2,070 & 11,906 & 10,292 & 3,499 & 3,080 & 11,805 & 12,455 \\
\hline 9 & 10,629 & 15,081 & 18,998 & 3,509 & 0,346 & 12,994 & 0,026 & 2,860 & 0,405 & 14,424 \\
\hline 11 & 8,451 & 36,486 & 1,157 & 5,733 & 7,573 & 31,862 & 1,414 & 5,892 & 7,659 & 23,834 \\
\hline 13 & 7,224 & 42,491 & 0,836 & 47,743 & 6,279 & 5,492 & 1,026 & 0,931 & 6,178 & 7,264 \\
\hline 15 & 6,283 & 31,145 & 6,740 & 3,420 & 0,340 & 27,038 & 0,011 & 3,675 & 0,394 & 24,257 \\
\hline 17 & 5,481 & 12,431 & 0,495 & 1,449 & 4,930 & 10,901 & 0,596 & 1,224 & 5,017 & 15,037 \\
\hline 19 & 4,939 & 4,095 & 0,400 & 0,562 & 4,250 & 3,575 & 0,481 & 0,423 & 4,148 & 4,977 \\
\hline 21 & 4,475 & 8,719 & 3,428 & 1,433 & 0,338 & 7,046 & 0,007 & 0,703 & 0,391 & 5,319 \\
\hline 23 & 4,060 & 5,023 & 0,277 & 0,750 & 3,670 & 4,623 & 0,326 & 0,441 & 3,756 & 7,155 \\
\hline 25 & 3,757 & 3,489 & 0,235 & 0,282 & 3,202 & 2,927 & 0,276 & 0,307 & 3,099 & 3,785 \\
\hline 27 & 3,481 & 5,917 & 2,067 & 0,739 & 0,337 & 4,784 & 0,007 & 0,381 & 0,390 & 3,647 \\
\hline 29 & 3,229 & 3,528 & 0,184 & 0,554 & 2,932 & 2,949 & 0,205 & 0,229 & 3,018 & 4,249 \\
\hline 31 & 3,034 & 1,036 & 0,157 & 0,194 & 2,562 & 1,253 & 0,181 & 0,086 & 2,457 & 2,058 \\
\hline 33 & 2,852 & 3,495 & 1,389 & 0,216 & 0,338 & 2,864 & 0,005 & 0,189 & 0,390 & 1,771 \\
\hline 35 & 2,683 & 4,140 & 0,127 & 0,251 & 2,448 & 3,417 & 0,141 & 0,158 & 2,532 & 1,875 \\
\hline 37 & 2,548 & 8,223 & 0,115 & 0,305 & 2,131 & 6,990 & 0,127 & 0,288 & 2,024 & 5,848 \\
\hline 39 & 2,418 & 11,888 & 0,996 & 4,037 & 0,338 & 2,014 & 0,004 & 0,123 & 0,390 & 0,924 \\
\hline $\begin{array}{c}\text { Действующее } \\
\text { значение }\end{array}$ & \multicolumn{2}{|c|}{$U_{1 \phi 1}, \mathrm{~B}$} & \multicolumn{2}{|c|}{$I_{1 \phi 1}, \mathrm{~A}$} & \multicolumn{2}{|c|}{$U_{2 \phi 1}, \mathrm{~B}$} & \multicolumn{2}{|c|}{$I_{2 \phi 1}, \mathrm{~A}$} & \multicolumn{2}{|c|}{$U_{2 л 1}$, В } \\
\hline 1-й гармоники & 520,864 & 447,176 & 66,013 & 52,615 & 134,816 & 115,820 & 201,675 & 173,191 & 234,969 & 208,547 \\
\hline THD, $\%$ & 39,839 & 13,442 & 212,766 & 11,802 & 0,561 & 5,457 & 0,190 & 2,807 & 0,682 & 7,566 \\
\hline
\end{tabular}

\begin{tabular}{|c|c|c|c|c|c|c|c|c|c|c|}
\hline Метод & 3 & 4 & 3 & 4 & 3 & 4 & 3 & 4 & 3 & 4 \\
\hline $\begin{array}{c}\text { Порядок } \\
\text { гармоники } k\end{array}$ & \multicolumn{2}{|c|}{$U_{1 \phi k}, \%$} & \multicolumn{2}{|c|}{$I_{1 \phi k}, \%$} & \multicolumn{2}{|c|}{$U_{2 \phi k}, \%$} & \multicolumn{2}{|c|}{$I_{2 \phi k}, \%$} & \multicolumn{2}{|c|}{$U_{2 л k}, \%$} \\
\hline 1 & 100,000 & 100,000 & 100,000 & 100,000 & 100,000 & 100,000 & 100,000 & 100,000 & 100,000 & 100,000 \\
\hline 3 & 27,982 & 21,530 & 150,616 & 108,047 & 0,608 & 1,371 & 0,444 & 0,582 & 0,643 & 1,392 \\
\hline 5 & 6,256 & 1,754 & 2,883 & 2,553 & 5,941 & 1,559 & 2,281 & 0,584 & 7,296 & 1,572 \\
\hline 7 & 5,819 & 3,859 & 14,402 & 10,252 & 1,213 & 2,441 & 0,315 & 0,675 & 1,202 & 1,674 \\
\hline 9 & 10,957 & 12,113 & 2,999 & 2,496 & 9,802 & 11,127 & 2,206 & 2,634 & 9,855 & 10,852 \\
\hline 11 & 15,727 & 20,930 & 3,420 & 1,851 & 13,847 & 19,178 & 2,602 & 3,732 & 13,773 & 19,923 \\
\hline 13 & 18,032 & 24,814 & 22,664 & 29,989 & 0,402 & 0,592 & 0,061 & 0,076 & 0,332 & 1,496 \\
\hline 15 & 16,740 & 20,995 & 1,433 & 2,602 & 15,225 & 18,137 & 2,106 & 2,522 & 13,491 & 18,785 \\
\hline 17 & 13,739 & 12,892 & 4,330 & 1,876 & 10,832 & 11,457 & 1,258 & 1,418 & 11,046 & 11,034 \\
\hline 19 & 11,138 & 8,431 & 6,770 & 3,465 & 6,481 & 5,415 & 0,691 & 0,581 & 6,263 & 5,516 \\
\hline 21 & 9,884 & 11,086 & 1,412 & 0,663 & 8,368 & 10,008 & 0,830 & 1,013 & 4,920 & 10,253 \\
\hline 23 & 7,986 & 10,490 & 5,123 & 6,166 & 1,400 & 1,613 & 0,168 & 0,133 & 1,732 & 1,547 \\
\hline 25 & 4,274 & 4,662 & 0,743 & 0,896 & 4,105 & 3,195 & 0,336 & 0,265 & 2,906 & 3,802 \\
\hline 27 & 2,509 & 3,591 & 1,155 & 0,532 & 0,870 & 3,070 & 0,065 & 0,262 & 1,036 & 4,077 \\
\hline 29 & 5,937 & 9,751 & 2,268 & 4,898 & 3,952 & 2,121 & 0,233 & 0,114 & 4,367 & 2,759 \\
\hline 31 & 7,690 & 11,313 & 0,351 & 0,668 & 7,248 & 10,023 & 0,420 & 0,628 & 9,749 & 13,390 \\
\hline 33 & 7,083 & 8,175 & 1,348 & 2,588 & 5,263 & 5,329 & 0,230 & 0,316 & 5,140 & 5,713 \\
\hline 35 & 5,699 & 2,601 & 1,730 & 0,533 & 1,228 & 1,787 & 0,040 & 0,065 & 0,717 & 2,315 \\
\hline 37 & 5,721 & 2,855 & 1,026 & 0,479 & 3,133 & 1,779 & 0,145 & 0,136 & 1,250 & 2,701 \\
\hline 39 & 6,226 & 5,360 & 1,503 & 1,472 & 4,679 & 1,710 & 0,202 & 0,108 & 4,246 & 0,709 \\
\hline $\begin{array}{c}\text { Действующее } \\
\text { значение }\end{array}$ & \multicolumn{2}{|c|}{$U_{1 \phi 1}, \mathrm{~B}$} & \multicolumn{2}{|c|}{$I_{1 \phi 1}, \mathrm{~A}$} & \multicolumn{2}{|c|}{$U_{2 \phi 1}, \mathrm{~B}$} & \multicolumn{2}{|c|}{$I_{2 \phi 1}, \mathrm{~A}$} & \multicolumn{2}{|c|}{$U_{2 л 1}$, В } \\
\hline 1-й гармоники & 504,700 & 492,928 & 63,204 & 63,956 & 129,690 & 126,670 & 193,041 & 189,136 & 229,134 & 219,677 \\
\hline THD, $\%$ & 28,666 & 22,187 & 150,624 & 108,057 & 4,719 & 2,192 & 0,487 & 0,592 & 4,295 & 1,562 \\
\hline
\end{tabular}


Гармонический состав полученных кривых напряжений и токов приведен в табл. 2, где также представлены значения суммарных коэффициентов гармонических составляющих THD, \%. Частота 1-й гармоники составляет 50 Гц. Частота несущего напряжения ШИМ 650 Гц.

\section{Заключение}

Подводя итог, можно констатировать, что предложена схема устройства питания вспомогательных цепей электровоза постоянного тока на основе трёхфазного полумостового АИН и трёхфазного Т со схемой соединения обмоток III/Y, обладающая следующими достоинствами: количество силовых транзисторов минимально, класс транзисторов по напряжению относительно низкий (допустимо использовать транзисторы с величиной рабочего напряжения 2,5 кВ с учетом возможности в контактной сети $U_{d}=4$ кВ), что удешевляет устройство. Стоимость комплекта полупроводниковых ключей типа HVIGBT [11] может быть снижена до 1,5-2,0 раза по сравнению с известными техническими решениями. Рекомендован лучший по совокупности энергетических характеристик, использованию входного напряжения и аспектам обеспечения электромагнитной совместимости метод управления ключами АИН - синусоидальная ШИМ. Методы формирования выходного напряжения предложенного устройства не отличаются от используемых в известных схемах, т.е. могут применяться отработанные технологии построения системы управления преобразователем.

\section{Лuтература}

1. Хоменко Б.И. Вспомогательные транзисторные преобразователи для перспективного ЭПС / Б.И. Хоменко, Г.И. Колпахчьян, И.В. Пехотский // Электровозостроение. $-2003 .-$ Т. 45. - С. 184-191.

2. Umezawa K. Power electronic devices for railway vehicles // FUJI Electric Review. - 2012. - Vol. 58, No. 4. PP. 175-181.

3. Macan M. Output DC voltage elimination in PWM converters for railway applications / M. Macan, I. Bahun, Z. Jakopovic // 17th int. conference on electrical drives and power electronics (EDPE 2011), The High Tatras, Slovakia 28-30 September, 2011. - Stará Lesná, 2011. - PP. 49-54.

4. Пустоветов М.Ю. Теоретическое исследование потенциала нейтральной точки нагрузки и токов утечки в тяговом асинхронном электроприводе электровоза постоянного тока // Изв. Транссиба. - 2012. - № 4(12). C. $116-122$.

5. Вольдек А.И. Электрические машины. - 2-е изд., перераб. и доп. - Л.: Энергия, 1974. - 840 с.
6. Бурков А.Т. Электронная техника и преобразователи. - М.: Транспорт, 1999. - 464 с.

7. Курочка А.А. Выбор алгоритма широтно-импульсной модуляции в автономном инверторе напряжения промышленного электровоза НПМ2 / А.А. Курочка, Д.А. Кабанов, Л.Д. Лушникова // Вестник ВЭлНИИ. - 2004. № 1. - С. 156-163.

8. Пустоветов М.Ю. Универсальная математическая модель трёхфазного трансформатора с единым магнитопроводом // Электротехника. - 2015. - № 2. - С. 57-60.

9. Пустоветов М.Ю. Математическая модель трёхфазного трансформатора // Изв. ТПУ. Инжиниринг георесурсов. - 2012. - Т. 321, №4. - С. 97-100.

10. Болотовский Ю.И. OrCAD 9.x. OrCAD 10.x. Практика моделирования / Ю.И. Болотовский, Г.И. Таназлы. - М.: СОЛОН-ПРЕСС, 2008. - 208 с.

11. Воронин П.А. Силовые полупроводниковые ключи. Семейства, характеристики, применение. - М.: ИД «Додэка-XXI», 2001. - 384 с.

\section{Пустоветов Михаил Юрьевич}

Канд. техн. наук, доцент каф. энергетики, автоматики и систем коммуникаций (ЭАСК)

Донского гос. технического ун-та (ДГТУ)

Тел.: +7-928-136-26-41

Эл. почта: mgsn2006@rambler.ru

Pustovetov M.Yu.

Power supply device for onboard auxiliary circuit of DC electric locomotive: scheme of the power part and methods to form the output voltage

An article provides a brief analysis of the circuits of semiconductor converters used to power the auxiliary circuits of DC electric locomotive and proposes the scheme of power part of the device that is different from the widely spread ones. The author investigates characteristics of the device in static mode. The device comprises a capacitive divider of input DC voltage of the three-phase half-bridge autonomous voltage source inverter and three-phase transformer with a specific winding connection scheme III/Y. A comparative consideration of the energy characteristics of the device at different methods of pulse width modulation is carried out. The use of catenary voltage, the efficiency, the harmonic composition of currents and voltages, the values of the total harmonic distortion and power factor were taken into account. Cost reduction for the set of power switches of the inverter up to $1.5-2.0$ times in comparison with the known technical solutions is achieved.

Keywords: autonomous voltage source inverter, three-phase transformer, DC electric locomotive, supply of auxiliary circuits, pulse width modulation, harmonic composition. 